\title{
Approaches, Tools and Applications for Sentiment Analysis Implementation
}

\author{
Alessia D'Andrea \\ Institute for Research on \\ Population and Social \\ Policies, National \\ Research Council \\ Via Palestro, 32, 00185, \\ Rome, Italy
}

\author{
Fernando Ferri \\ Institute for Research on \\ Population and Social \\ Policies, National \\ Research Council \\ Via Palestro, 32, 00185, \\ Rome, Italy
}

\author{
Patrizia Grifoni \\ Institute for Research on \\ Population and Social \\ Policies, National \\ Research Council \\ Via Palestro, 32, 00185, \\ Rome, Italy
}

\author{
Tiziana Guzzo \\ Institute for Research on \\ Population and Social \\ Policies, National \\ Research Council \\ Via Palestro, 32, 00185, \\ Rome, Italy
}

\begin{abstract}
The paper gives an overview of the different sentiment classification approaches and tools used for sentiment analysis. Starting from this overview the paper provides a classification of (i) approaches with respect to features/techniques and advantages/limitations and (ii) tools with respect to the different techniques used for sentiment analysis. Different application fields of application of sentiment analysis such as: business, politic, public actions and finance are also discussed in the paper.
\end{abstract}

\section{Keywords}

Sentiment analysis, Social Media, Machine-learning approach, Lexicon-based approach, Sentiment classification

\section{INTRODUCTION}

People share knowledge, experiences and thoughts with the world by using Social Media like blogs, forums, wikis, review sites, social networks, tweets and so on. This has changed the manner in which people communicate and influence social, political and economic behavior of other people in the Web 2.0. Indeed the Web 2.0 allows everyone having a voice, promising to boost human collaboration capabilities on a worldwide scale, enabling individuals to share opinions by means of read-write Web and user's generated contents. According to [1] an opinion "is simply a positive or negative sentiment, view, attitude, emotion, or appraisal about an entity or an aspect of the entity" from an opinion holder at a specific time $[2 ; 3]$. The entity can be a product/service, event, person, organization, or topic consisting of aspects (features/attributes) that represents both components and attributes of the entity.

With the explosion of user generated opinions there is the need by companies, politicians, service providers, social psychologists, researchers and other actors to analyze them in order to implement better decision choices.

The term sentiment analysis first appeared in [4], however the research on sentiments/opinions appeared earlier $[5 ; 6 ; 7 ; 8$; 9]. The literature on sentiment analysis focused on different domains, from management sciences to computer science, social sciences and business due to its importance to society as whole and different tasks such as: subjective expressions

[10], sentiments of words [11], subjective sentences [12], and topics $[4 ; 13 ; 14)$
The sentiment analysis is a complex process that involves 5 different steps to analyze sentiment data. These steps are:

- data collection: the first step of sentiment analysis consists of collecting data from user generated content contained in blogs, forums, social networks. These data are disorganized, expressed in different ways by using different vocabularies, slangs, context of writing etc. Manual analysis is almost impossible. Therefore, text analytics and natural language processing are used to extract and classify;

- text preparation: consists in cleaning the extracted data before analysis. Non-textual contents and contents that are irrelevant for the analysis are identified and eliminated;

- sentiment detection: the extracted sentences of the reviews and opinions are examined. Sentences with subjective expressions (opinions, beliefs and views) are retained and sentences with objective communication (facts, factual information) are discarded

- sentiment classification: in this step, subjective sentences are classified in positive, negative, good bad; like, dislike, but classification can be made by using multiple points;

- presentation of output: the main objective of sentiment analysis is to convert unstructured text into meaningful information. When the analysis is finished, the text results are displayed on graphs like pie chart, bar chart and line graphs. Also time can be analysed and can be graphically displayed constructing a sentiment time line with the chosen value (frequency, percentages, and averages) over time.

The paper provides an overview of studies on the sentiment classification step; In particular starting from this overview the paper a classification of (i) sentiment classification approaches with respect to features/techniques and advantages/limitations and (ii) tools with respect to the different techniques used for sentiment analysis. Different application fields of sentiment analysis such as: business, politic, public actions and finance are also discussed in the paper.

The sentiment classification approaches can be classified in: (i) machine learning (ii) lexicon based and (iii) hybrid 
approach. The machine learning approach is used for predicting the polarity of sentiments based on trained as well as test data sets.

While the lexicon based approach does not need any prior training in order to mine the data. It uses a predefined list of words, where each word is associated with a specific sentiment. Finally in the hybrid approach, the combination of both the machine learning and the lexicon based approaches has the potential to improve the sentiment classification performance. On considering the tools used for sentiments analysis, the most used tools for detecting the feelings polarity are Emoticons, LIWC, SentiStrengh, Senti WordNet, SenticNet, Happiness Index, AFINN, PANAS-t, Sentiment140, NRC, EWGA and FRN.

Sentiment analysis is used mainly in different fields such as marketing, political and sociological.

In marketing field companies use it to develop their strategies, to understand customers' feelings towards products or brand how people respond to their campaigns or product launches and why consumers don't buy some products. In political field, it is used to track of political view, to detect consistency and inconsistency between statements and actions at the government level; it can be used to predict election results. Sentiment analysis also is used to monitor and analyse social phenomena, for the spotting of potentially dangerous situations and determining the general mood of the blogosphere. The sentiment analysis then represents an important element for any subject (policy makers, stakeholders, companies etc.) to perform different kinds of activities such as: predict financial performance [15], understand consumers' perception [16] provide early warnings [17; 18], define election outcomes etc. In all of these examples, the sentiment input is whether a given consumer opinion has negative, positive or neutral polarity regarding the different target of interest [19]. The large amount of these contents required the use of automated techniques for analyzing since manually it is not possible. According to [20], researchers have found ways to avoid the use of manual annotation by utilizing existing online textual content generated from sites such as Epinion, Amazon, Rotten Tomatoes, Twitter, and Facebook.

Starting from these considerations Section 2 gives an overview of different studies provided in the literature on sentiment analysis domain. Section 3 provides a classification of: (i) sentiment classification approaches with respect to features/techniques and advantages and limitations (ii) tools for sentiment analysis with respect to the different techniques used for sentiment analysis. Finally, section 4 concludes the paper.

\section{BACKGROUND}

Sentiment analysis is a new field of research born in Natural Language Processing (NLP), aiming at detecting subjectivity in text and/or extracting and classifying opinions and sentiments. Sentiment analysis studies people's sentiments, opinions, attitudes, evaluations, appraisals and emotions towards services, products, individuals, organizations, issues, topics events and their attributes [20].

In sentiment analysis text is classified according to the following different criteria:

- the polarity of the sentiment expressed (into positive, negative, and neutral);

- the polarity of the outcome (e.g. improvement versus death in medical texts) [21];
- $\quad$ agree or disagree with a topic (e.g. political debates) [22];

- $\quad$ good or bad news [23];

- $\quad$ support or opposition [24; 25];

- $\quad$ pros and cons [26].

For the sentiment analysis implementation different sentiment classification approaches and tools are used. In the following sections an overview of them is given.

\subsection{Sentiment classification approaches}

The Sentiment classification is a task of classifying a target unit in a document to positive (favorable) or negative (unfavorable) class. There are three main classification levels [27]:

- document level: classifies an opinion document as expressing a positive or negative opinion or sentiment. It considers the whole document a basic information unit (talking about one topic);

- sentence-level: classifies sentiment expressed in each sentence. If the sentence is subjective it classifies it in positive or negative opinions;

- aspect-level: classifies the sentiment with respect to the specific aspects of entities. Users can give different opinions for different aspects of the same entity.

At document level it is possible to classify whether a whole users opinion expresses a positive or negative sentiment. For example, given a product/service review, it is possible to determine whether the review expresses an overall positive or negative opinion about the product/service. The sentence determines whether each sentence expresses a positive or negative. While the entity/aspect level, instead of looking at language construction (sentences, phrases, paragraphs, clauses etc), directly focus on the opinion itself. It is based on the idea that an opinion consists of a sentiment (positive or negative) and a target (of opinion). The document sentiment classification approach is used by [6] that classify movie reviews by using supervised machine learning method. In [28] the authors used the semantic orientation of words defined by [8] and several information from the Web and thesaurus. They achieved $85 \%$ accuracy with and the semantic orientation of words and the lemmatized word unigram. While the study provided by [29] used the sentence level classification approach. It considered word dependency trees as features for sentence-wise sentiment polarity classification. On the contrary the study conducted by [8] determined the relationship between a polarity-unknown word and a set of selected manually seeds for classifying the polarity-unknown word into positive or negative class. Also the study provided by [11] to extract sentiment polarities by using expressions such as or "fast but inaccurate" or "beautiful and smart".

In [30] a survey on different methods of sentiment analysis available in literature related to product reviews (such as machine learning, semantic orientation, opinion polling, holistic lexicon-based approach etc.) is carried out. The survey underlines that sentiment analysis/opinion mining play vital role to make decision about product /services. Another survey on approaches used for sentiment analysis is provided in [31] in which three approaches for performing sentiment extraction are described:

- $\quad$ subjective lexicon approach: is a list of words to witch is assigned a score that indicates its nature in terms of positive, negative or objective; 
- n-gram modeling approach: that can use uni-gram, bi-gram, tri-gram or combination of these for the sentiments classification;

- machine learning approach: performs the semi and/or supervised learning through the extraction of the features from the text and learn the model.

While [32] analyse three types of techniques for Sentiment Classification: (i) machine learning approach, (ii) lexiconbased approach and (iii) hybrid approach. The machine learning approach is used for predicting the polarity of sentiments based on trained as well as test data sets. It applies the ML algorithms and uses linguistic features. The main advantage of this method is the ability to adapt and create trained models for specific purposes and contexts, its main disadvantage is the low applicability of the method on new data because is necessary the availability of labeled data that could be costly or even prohibitive. It can use supervised and unsupervised methods. The machine learning uses a supervised approach when there is a finite set of classes (positive and negative). This method needs labeled data to train classifiers [6]. In a machine learning based classification a training set is used by an automatic classifier to learn the different characteristics of documents, and a test set is used to validate the performance of the automatic classifier. The unsupervised methods are used when it is difficult to find labeled training documents. Unsupervised learning does not require prior training in order to mine the data. Unsupervised approaches to document-level sentiment analysis are based on determining the semantic orientation (SO) of specific phrases within the document. If the average SO of these phrases is above some predefined threshold the document is classified as positive, otherwise it is deemed negative. Among the machine learning approaches the most used are: (i) Bayesian Networks: it is a probabilistic approach that models relationships between features in a very general way. It is based on directed acyclic graph in which nodes are variables and arcs represent the dependence between variables (ii) Naive Bayes Classification: it is an approach particularly suited when the dimensionality of the inputs is high. Despite its simplicity, it can often outperform more sophisticated classification methods (iii) Maximum Entropy: this method is mostly used as alternatives to Naive Bayes classifiers because it does not assume statistical independence of the random variables (features) that serve as predictors. The principle behind Maximum Entropy is to find the best probability distribution among prior test data. (iv) Neural Networks: this model is based on a collection of natural/artificial neurons uses for mathematical and computational model analysis (v) Support Vector Machine: it is a supervised learning model which analyzes data and patterns that can be used for classification and regression analysis. The basic idea behind this is to find a maximum margin hyper plane represented by vector. It finds an optimal solution. While the lexicon-based approach does not need any prior training in order to mine the data. It uses a predefined list of words, where each word is associated with a specific sentiment. They are based on the counting of positive and negative words. These methods vary according to the context in which they were created. Lexical don't need labeled data, but is hard to create a unique lexical-based dictionary to be used for different contexts. For example slang used in Social Networks is rarely supported in lexical methods [33].
Among the lexicon-based approaches the most used are: (i) Dictionary based approach: it is a method that translates a word by word as a dictionary without correlating the meaning of words between them

(ii) Novel Machine Learning Approach: it integrates important linguistic features into automatic learning (iii) Corpus based approach: it has been widely used to explore both written and spoken texts in order to assign a sentiment factor of words that depend on frequency of their occurrences (iv) Ensemble Approaches in sentiment classification: it increases classification accuracy by combining arrays of specialized learners.

The study provided by [34] gives an example of the lexicon based approach applied on a morphologically rich language: Urdu It focuses on the sentence grammatical structures, besides to the morphological structure of the words. For the analysis, two types of grammatical structures (adjective phrases as Senti-Units and nominal phrases as their targets) are extracted and then linked. For the extraction and linking two parsing methods have been implemented: shallow and dependency parsing. In [35] the authors focused on the lexicon-based approach for Arabic sentiment analysis by building the main two components of the lexicon-based sentiment analysis approach: the lexicon and the sentiment analysis tool. The study provided a guide for the researchers in their on-going efforts to improve lexicon-based sentiment analysis.

\subsection{Tools for sentiment analysis}

There are many studies that provide methods and tools used for sentiments analysis. The most used tools for detecting the feelings polarity (negative and positive affect) of a message is based on the emoticons. Emoticons are face-based and symbolize sad or happy feelings, although there are a wide range of non-facial variations. To extract the feelings polarity from emoticons, different set of common emoticons can be used (http://messenger.msn.com/Resource/Emoticons.aspx; http://www.cool-smileys.com/text-emoticons;

http://messenger.yahoo.com/features/emoticons). Therefore, emoticons have been often used in combination with other techniques for building a training dataset in supervised machine learning techniques [36]. Another method is the Linguistic Inquiry and Word Count [37] that allows analysing not only positive and negative but also emotional, cognitive, and structural components of a text based on the use of a dictionary containing words and their classified categories. For example, the word "agree" belongs to the word categories: assent, affective, positive emotion, positive feeling, and cognitive process. This software is available at http://www.liwc.net/. Happiness Index [38] is a sentiment scale that uses the popular Affective Norms for English Words (ANEW) [39]. It gives scores for a given text between 1 and 9, indicating the amount of happiness. The authors calculated the frequency that each word from the ANEW appears in the text and then computed a weighted average of the valence of the ANEW study words. Another tool is the SentiStrength (http://sentistrength.wlv.ac.uk/Download) that is considered by [40] "the most popular stand-alone sentiment analysis tool". It uses a sentiment lexicon for assigning scores to negative and positive phrases in text. For identifying the feeling polarity several key classifiers are proposed [41, 42]. In [43] the SentiWordNet (at http://sentiwordnet.isti.cnr.it/.) tool is described. SentiWordNet is a lexical resource publicly available for supporting sentiment classification and opinion mining applications. It is based on an English lexical dictionary called WordNet [44] that ghaters adjectives, nouns, verbs etc. into synonym sets called synsets. 
Each synset is associated to three numerical scores Pos(s), $\mathrm{Neg}(\mathrm{s})$, and Obj(s) which indicate how positive, negative, and "objective" (neutral) the terms contained in the synset are. The scores, which are in the values of $[0,1]$ and add up to 1 , are obtained using a semi-supervised machine learning method. The tool, used in opinion mining, is based on WordNet an English lexical dictionary that collect nouns, verbs, adjectives and other grammatical classes into synonym sets (synsets) [44]. Another tool is the PANAS-t [45]. The tool consists of an adapted version of the Positive Affect Negative Affect Scale (PANAS) [46], method used in psychology. The PANAS-t tracks increases or decreases in sentiments over time; it is based on a large set of words associated with eleven moods: joviality, assurance, serenity, surprise, fear, sadness, guilt, hostility, shyness, fatigue, and attentiveness. This method computes the score for each sentiment for a given time period as values between $[-1.0$, 1.0] to indicate the change. The open source tool SailAil Sentiment Analyzer (SASA) [47] was evaluated with 17,000 labeled tweets on the 2012 U.S. Elections. It was evaluated also by the Amazon Mechanical Turk (AMT), where "turkers" were invited to label tweets as positive, negative, neutral, or undefined. The SASA python package version 0.1 .3 is available at https://pypi.python.org/pypi/sasa/0.1.3. In [45] the authors developed a new sentiment analysis method that combines the different described approaches in order to provide the best coverage and competitive agreement. They implemented a public Web API, called iFeel (http://www.ifeel.dcc.ufmg.br), which provides comparative results among the different sentiment methods for a given text. In [48], the authors described SenticNet a tool that explores artificial intelligence and semantic Web techniques. The tool explores artificial intelligence and semantic Web techniques. It uses Natural Language Processing (NLP) techniques to infer the polarity of common sense concepts from natural language text at a semantic level, rather than at the syntactic level. SenticNet was tested to measure the level of polarity in opinions of patients about the National Health Service in England [48]. SenticNet version 2.0 is available at http://sentic.net/. In $[49,50]$ EWGA and FRN tools are used. The EWGA tool uses an entropy-weighted genetic algorithm for an efficiently selection of features for sentiment classification using a wrapper-model. While the FRN uses a feature relation network considering two syntactic n-gram relations: parallel relations and subsumption [51]. Sentiment140 formerly known as Twitter Sentiment discovers the positive and negative opinions and sentiment of a brand, product, or topic on Twitter. This tool uses classifiers built from machine learning algorithms. Unlike other tools that show aggregated numbers which makes it difficult to assess how accurate their classifiers are, this tool is able to classify individual tweets. The NRC Hashtag Sentiment Lexicon (version 0.1) is a list of words with associations to positive and negative sentiments. The lexicon is distributed in three files: unigrams-pmilexicon.txt, bigrams-pmilexicon.txt, and pairs-pmilexicon.txt. The NRC Emotion Lexicon is comprised of frequent English nouns, verbs, adjectives, and adverbs annotated for eight emotions (joy, sadness, anger, fear, disgust, surprise, trust, and anticipation) as well as for positive and negative sentiment.

\section{CLASSIFICATION OF SENTIMENT ANALYSIS APPROACHES}

Starting from the analysis provided in the previous sections a classification of sentiment analysis approaches with respect to features/techniques and advantages /limitations is provided in Table 1.
Table 1: Sentiment classification approaches

\begin{tabular}{|c|c|c|c|}
\hline \multicolumn{2}{|c|}{$\begin{array}{c}\text { SENTIMENT } \\
\text { CLASSIFICATION } \\
\text { APPROACHES }\end{array}$} & $\begin{array}{l}\text { FEATURES/ } \\
\text { TECNIQUES }\end{array}$ & $\begin{array}{l}\text { ADVANTAGES } \\
\text { AND } \\
\text { LIMITATIONS }\end{array}$ \\
\hline $\begin{array}{l}\text { Machine } \\
\text { learning }\end{array}$ & $\begin{array}{c}\text { Bayesian } \\
\text { Networks } \\
\text { Naive Bayes } \\
\text { Classification } \\
\text { Maximum } \\
\text { Entropy } \\
\text { Neural } \\
\text { Networks } \\
\text { Support } \\
\text { Vector } \\
\text { Machine }\end{array}$ & $\begin{array}{l}\text { Term presence } \\
\text { and frequency } \\
\text { Part of speech } \\
\text { information } \\
\text { Negations } \\
\text { Opinion words } \\
\text { and phrases }\end{array}$ & $\begin{array}{l}\text { ADVANTAGES } \\
\text { the ability to } \\
\text { adapt and create } \\
\text { trained models } \\
\text { for specific } \\
\text { purposes and } \\
\text { contexts } \\
\text { LIMITATIONS } \\
\text { the low } \\
\text { applicability to } \\
\text { new data because } \\
\text { it is necessary the } \\
\text { availability of } \\
\text { labeled data that } \\
\text { could be costly } \\
\text { or even } \\
\text { prohibitive }\end{array}$ \\
\hline $\begin{array}{c}\text { Lexicon } \\
\text { based }\end{array}$ & $\begin{array}{l}\text { Dictionary } \\
\text { based } \\
\text { approach } \\
\text { Novel } \\
\text { Machine } \\
\text { Learning } \\
\text { Approach } \\
\text { Corpus based } \\
\text { approach } \\
\text { Ensemble } \\
\text { Approaches }\end{array}$ & $\begin{array}{c}\text { Manual } \\
\text { construction, } \\
\text { Corpus-based } \\
\begin{array}{c}\text { Dictionary- } \\
\text { based }\end{array}\end{array}$ & $\begin{array}{l}\text { ADVANTAGES } \\
\text { wider term } \\
\text { coverage } \\
\text { LIMITATIONS } \\
\text { finite number of } \\
\text { words in the } \\
\text { lexicons and the } \\
\text { assignation of a } \\
\text { fixed sentiment } \\
\text { orientation and } \\
\text { score to words }\end{array}$ \\
\hline Hybrid & $\begin{array}{c}\text { Machine } \\
\text { learning } \\
\text { Lexicon based }\end{array}$ & $\begin{array}{c}\text { Sentiment } \\
\text { lexicon } \\
\text { constructed } \\
\text { using public } \\
\text { resources for } \\
\text { initial sentiment } \\
\text { detection } \\
\text { Sentiment } \\
\text { words as } \\
\text { features in } \\
\text { machine } \\
\text { learning method }\end{array}$ & $\begin{array}{l}\text { ADVANTAGES } \\
\text { lexicon/learning } \\
\text { symbiosis, the } \\
\text { detection and } \\
\text { measurement of } \\
\text { sentiment at the } \\
\text { concept level } \\
\text { and the lesser } \\
\text { sensitivity to } \\
\text { changes in topic } \\
\text { domain } \\
\text { LIMITATIONS } \\
\text { noisy reviews }\end{array}$ \\
\hline
\end{tabular}

Machine learning based approach uses classification technique to classify text; it consists of two sets of documents: training and a test set. The training set is used for learning the differentiating characteristics of a document, while the test set is used for checking how well the classifier performs. The features of machine learning based approach for sentiment classification are:

- term presence and their frequency: that includes uni-grams or n-grams and their presence or frequency.

- $\quad$ part of speech information: used for disambiguating sense which is used to guide feature selection 
- negations: has the potential of reversing sentiments opinion words/phrases: that expresses positive or negative sentiments.

The lexicon based approach uses sentiment dictionary with opinion words and match them with the data for determining polarity. There are three techniques to construct a sentiment lexicon: manual construction, corpus-based methods and dictionary-based methods. The manual construction is a difficult and time-consuming task. Corpus-based methods can produce opinion words with relatively high accuracy. Finally, in the dictionary based techniques, the idea is to first collect a small set of opinion words manually with known orientations, and then to grow this set by searching in the WordNet dictionary for their synonyms and antonyms.

Finally, in the hybrid approach, the combination of both the machine learning and the lexicon based approaches has the potential to improve the sentiment classification performance. There are some advantages and limitations in using these different approaches depending on the purpose of the analysis. We provide an overview of the main. The main advantage of machine learning approaches is the ability to adapt and create trained models for specific purposes and contexts, while the limitation is that it is difficult integrating into a classifier, general knowledge which may not be acquired from training data. Furthermore, learnt models often have poor adaptability between domains or different text genres because they often rely on domain specific features from their training data. Lexicon-based approaches have the advantage that general knowledge sentiment lexicons have wider term coverage, however these approaches have two main limitations. Firstly, the number of words in the lexicons is finite, which may constitute a problem when extracting sentiment from very dynamic environments. Secondly, sentiment lexicons tend to assign a fixed sentiment orientation and score to words, irrespective of how these words are used in a text.

The main advantages of hybrid approaches are the lexicon/learning symbiosis, the detection and measurement of sentiment at the concept level and the lesser sensitivity to changes in topic domain. While the main limitation is that reviews are with a lot of noise (irrelevant words for the subject of the review) are often assigned a neutral score because the method fails to detect any sentiment.

Moreover a classification of tools for sentiment analysis according to the different techniques used for sentiment analysis is given in Table 2 .

Table 2. Tools for sentiment analysis

\begin{tabular}{|c|c|}
\hline $\begin{array}{c}\text { TOOLS FOR } \\
\text { SENTIMENT ANALYSIS }\end{array}$ & $\begin{array}{c}\text { TECNIQUES USED BY } \\
\text { TOOLS }\end{array}$ \\
\hline EMOTICONS & $\begin{array}{c}\text { Emoticons contained in the } \\
\text { text }\end{array}$ \\
\hline LIWC & $\begin{array}{c}\text { Dictionary and sentiment } \\
\text { classified categories }\end{array}$ \\
\hline SentiStrengh & $\begin{array}{c}\text { LIEC dictionary with new } \\
\text { features to strength and weak } \\
\text { sentiments }\end{array}$ \\
\hline Senti WordNet & $\begin{array}{c}\text { Lexical dictionary and } \\
\text { scores obtained by semi- } \\
\text { machine learning approaches }\end{array}$ \\
\hline SenticNet & $\begin{array}{c}\text { Natural language processing } \\
\text { approach for inferring the } \\
\text { polarity at semantic level }\end{array}$ \\
\hline Happiness Index & $\begin{array}{c}\text { Affective Norms for English } \\
\text { Words (ANEW) and scores }\end{array}$ \\
\hline
\end{tabular}

\begin{tabular}{|c|c|}
\hline & $\begin{array}{l}\text { for evaluating happiness in } \\
\text { the text }\end{array}$ \\
\hline AFINN & $\begin{array}{c}\text { Affective Norms for English } \\
\text { Words (ANEW) but more } \\
\text { focused on the language } \\
\text { used in microblogging } \\
\text { platforms. }\end{array}$ \\
\hline PANAS- $\mathrm{t}$ & $\begin{array}{c}\text { Eleven-sentiment } \\
\text { psychometric scale }\end{array}$ \\
\hline Sentiment 140 & $\begin{array}{c}\text { API that allows classifying } \\
\text { tweets to polarity classes } \\
\text { positive, negative and } \\
\text { neutral. }\end{array}$ \\
\hline NRC & $\begin{array}{c}\text { Large set of human-provided } \\
\text { words with their emotional } \\
\text { tags. }\end{array}$ \\
\hline EWGA & $\begin{array}{c}\text { Entropy-weighted genetic } \\
\text { algorithm }\end{array}$ \\
\hline FRN & $\begin{array}{c}\text { Feature relation network } \\
\text { considering syntactic n-gram } \\
\text { relations }\end{array}$ \\
\hline
\end{tabular}

The different approaches and tools analysed in this paper can be applied in different fields such as: business, politic, public actions and finance. On considering the business field many studies have been developed in the area of reviews of consumer products and services. There are many websites that provide automated summaries of reviews about products, such as Google Product Search. In [47], the authors developed SumView, a Web-based system that summarizes the product reviews and customer opinions. It integrates review crawling from Amazon.com, automatic product feature extraction along with a text field where users can input their desired features, and sentence selection using the proposed feature-based weighted non-negative matrix factorization algorithm. The most representative sentences are selected to form the summary for each product feature [47]. In the business domain context, sentiment analysis is also used for brand reputation, online advertising and on-line commerce. Sentiment analysis is used to monitor the reputation of a specific brand on Twitter and/or Facebook. Tweetfeel is an application that performs real-time analysis of tweets that contain a given term (http://www.tweetfeel.com). With respect to on-line advertising, it has become one of the major revenue sources of the Web ecosystem. In this context, recent applications of sentiment analysis are in Blogger Centric Contextual Advertising [52] and dissatisfaction oriented online advertising [53] which refer to the development of personal ads in any blog page, chosen in according to company business interests. Another application of sentiment analysis in the business domain is represented by the on-line commerce. The assumption is that consumers value others' opinions about restaurants, travel and stores guide each consumer in searching, hence Bing- and Google-computed star ratings. An important study in this context is developed by [54] that provided a Senti-lexicon for restaurant reviews. With respect to the politic domain, the voting advise applications represent an important application of sentiment analysis. It enables campaign managers to track how voters feel about different issues and how they relate to the speeches and actions of the candidates. An analysis of tweets related to the 2010 campaign can be found at ttp://www.nytimes.com/interactive/us/politics/2010-twittercandidates.html. In this context, sentiment analysis is also used to clarify politicians' positions, such as what public figures support or oppose, to enhance the quality of information that voters have access to [24]. 
Sentiment analysis is also used within the public actions implementation process. In this context sentiment analysis gives an important contribution also in monitoring real-world events.

Linguists at UT Austin use sentiment analysis to get a read on the rapidly evolving situation in the middle east. In particular sentiment analysis for monitoring critical information about earthquake locations and magnitude, riot locations; the monitoring help policy makers to minimise damage in areas which are expected to be affected next by such events. Another important application of sentiment analysis is the monitoring of the opinions that people submit about pending policy or government-regulation proposals $[55,56]$ and legal matters sometimes also known as "blawgs" [57]. A new emerging sentiment analysis domain is represented by the modern intelligent transportation systems (ITSs). For the completeness of ITS space, it is necessary to collect and analyze the public opinions exchange. The first attempt to apply sentiment analysis on the area of traffic is represented by the study developed by [58] where a traffic sentiment analysis (TSA) has been developed. The TSA allows analysing the traffic problem in a humanizer way. Finally, with respect to the finance domain, millions of financial news are circulating daily on the Web and financial markets are continuously changing and growing. There are numerous news items, articles, blogs, and tweets about each public company. A sentiment analysis system can use them and aggregate the sentiment about them as a single score that can be used by an automated trading system, such as the system The Stock Sonar (http://www.thestocksonar.com) that shows graphically the daily positive and negative sentiment about each stock alongside the graph of the price of the stock. In [59], the authors have investigated the Arizona Financial Text (AZFinText) system a financial news article prediction system, and has paired it with a sentiment analysis tool. They have found that the financial news articles have a direct impact on influencing the prices of commodities and shares. The analysis of finance news and opinions also allow monitoring the financial risk. The study conducted by [47] uses the finance- specific sentiment lexicon to model the relations between sentiment information and financial risk. In specific, they formulate the problem as two different prediction tasks: regression and ranking.

In Table 3 a schematization of sentiment analysis applications is provided.

Table 3: Sentiment analysis applications

\begin{tabular}{|l|}
\hline \multicolumn{1}{|c|}{ SENTIMENT ANALYSIS APPLICATIONS } \\
\hline \multicolumn{1}{|c|}{ BUSINESS } \\
\hline Consumers voice \\
\hline Brand reputation \\
\hline $\begin{array}{l}\text { Online advertising: } \\
\text { Blogger Centric Contextual Advertising } \\
\text { Dissatisfaction oriented online advertising }\end{array}$ \\
\hline On-line commerce \\
\hline \multicolumn{1}{|c|}{ POLITIC } \\
\hline Voting advise applications \\
\hline Clarification of politicians' positions \\
\hline \multicolumn{2}{|c|}{ PUCTIONS } \\
\hline Real-world events monitoring \\
\hline Legal matters "blawgs" \\
\hline Policy or government-regulation proposals \\
\hline Intelligent transportation systems \\
\hline
\end{tabular}

FINANCE

Prices of commodities and shares evolution

Financial risk individuation

\section{CONCLUSION}

The paper starting from the analysis of different studies provided in the literature, provides a classification of (i) sentiment classification approaches with respect to features/techniques and advantages /limitations (ii) tools for sentiment analysis with respect to the different techniques used for sentiment analysis.

The sentiment classification approaches can be classified in (i) machine learning (ii) lexicon based and (iii) hybrid approach. The machine learning approach is used for predicting the polarity of sentiments based on trained as well as test data sets. While the lexicon based approach does not need any prior training in order to mine the data. It uses a predefined list of words, where each word is associated with a specific sentiment. Finally in the hybrid approach, the combination of both the machine learning and the lexicon based approaches has the potential to improve the sentiment classification performance.

On considering the tools used for sentiments analysis, the most used tools for detecting the feelings polarity (negative and positive affect) are discussed in the paper: Emoticons, LIWC, SentiStrengh, Senti WordNet, SenticNet, Happiness Index, AFINN, PANAS-t, Sentiment140, NRC, EWGA and FRN.

The different approaches and tools analysed in the paper can be applied in different fields such as: business, politic, public actions and finance. In the business domain sentiment analysis is mainly used for detecting consumer's voice, brand reputation and the online advertising and on-line commerce trend. With respect to the politic domain, the voting advise represents an important application of sentiment analysis that is also used to clarify politicians' positions enhancing the quality of information that voters have access to. Sentiment analysis is also used within the public actions implementation process. In this context it gives an important contribution in monitoring real-world events. Another important application of sentiment analysis is the monitoring of the opinions that people submit about pending policy or government-regulation proposals and legal matters. A new emerging sentiment analysis domain is also represented by the modern intelligent transportation systems. Finally with respect to the finance domain, the sentiment analysis is used to detect the trend of prices of commodities and shares evolution and the financial risks.

A future challenge in applying sentiment classification approaches and tools for sentiment analysis of posts in social media is to overcome the ambiguity that actually represents particular problem since it is not easily make use of coreference information. Typically the analysed posts contain irony and sarcasm, which are particularly difficult to detect. So an evolution of approaches and tools is required to overcome this limitation.

\section{REFERENCES}

[1] Liu, B. 2006. Web Data Mining: Exploring Hyperlinks, Contents, and Usage Data, Springer.

[2] Bethard, S., Hong, Y., Thornton, A., Hatzivassiloglou, V., Jurafsky, D. 2004. Automatic extraction of opinion propositions and their holders. In Proceedings of the 
AAAI Spring Symposium on Exploring Attitude and Affect in Text.

[3] Wiebe, J. \& Riloff, E. 2005. Creating subjective and objective sentence classifiers from unannotated texts. Computational Linguistics and Intelligent Text Processing, 2005, pp. 486-497.

[4] Nasukawa ,T. \& Yi, J. 2003. Sentiment analysis: capturing favorability using natural language processing. In Proceedings of the 2nd international conference on Knowledge capture, October 23-25, 2003. (pp. 70-77). Florida, USA.

[5] Morinaga, S., Yamanishi, K., Tateishi, K., Fukushima, T. 2002. Mining product reputations on the web. In Proceedings of the eighth ACM SIGKDD international conference on Knowledge discovery and data mining, pp. 341-349.

[6] Pang, B., Lee, L., Vaithyanathan, S. 2002. Thumbs up? Sentiment Classification using Machine Learning Techniques. Proc. of 7th EMNLP, pp.79-86.

[7] Tong, R.M. 2001. An operational system for detecting and tracking opinions in on-line discussion. In Proceedings of SIGIR Workshop on Operational Text Classification.

[8] Turney, P. 2002. Thumbs up or thumbs down? semantic orientation applied to unsupervised classifcation of reviews. In Proceedings of the 40th ACL, pp. 417-424.

[9] Wiebe, J. (2000) Learning subjective adjectives from corpora. In Proceedings of National Conference on Artificial Intelligence.

[10] Wilson, T., Wiebe, J., Hoffmann, P. 2009. Recognizing contextual polarity: An exploration of features forphraselevel sentiment analysis. Computational Linguistics, 35(3), pp. 399-433.

[11] Hatzivassiloglou, V. \& McKeown, K.R. 1997. Predicting the semantic orientation of adjectives. In Proceedings of the 8th conference on European chapter of the association for computationnal linguistics Madrid, Spain, pp.174-181.

[12] Pang, B., \& Lee, L. 2004. A sentimental education: sentiment analysis using subjectivity summarization based on minimum cuts. In Proceedings of the 42nd annual meeting of the Association for Computational Linguistics (ACL), pp. 271-278. Barcelona, Spain

[13] Yi, J., Nasukawa, T., Niblack, W., Bunescu, R. 2003. Sentiment analyzer: extracting sentiments about a given topic using natural language processing techniques. In Proceedings of the 3rd IEEE international conference on data mining (ICDM 2003), November 19-22, 2003, pp. 427-434 Florida, USA.

[14] Hiroshi, K., Tetsuya, N., Hideo, W. 2004. Deeper sentiment analysis using machine translation technology. In Proceedings of the 20th international conference on computational linguistics (COLING 2004), August 2327, pp. 494-500, Geneva, Switzerland.

[15] Bollen, J., Mao, H., Zeng, X. 2011. Twitter mood predicts the stock market. Journal of Computational Science, 2(1), pp. 1-8.

[16] Smith, A.N., Fischer, E., Yongjian, C. 2012. How does brand-related user-generated content differ across across
YouTube, Facebook, and Twitter? Journal of Interactive Marketing, 26(2), pp. 102-113.

[17] Abbasi, A., Fu, T., Zeng, D., Adjeroh, D. 2013. Crawling Credible Online Medical Sentiments for Social Intelligence. Proceedings of the ASE/IEEE International Conference on Social Computing.

[18] Fu, T., Abbasi, A., Zeng, D., Chen, H. 2012. Sentimental Spidering: Leveraging Opinion Information in Focused Crawlers. ACM Transactions on Information Systems, 30(4), 24.

[19] Abbasi, A., Chen, H., Salem, A. 2008. Sentiment Analysis in Multiple Languages: Feature Selection for Opinion Classification in Web Forums. ACM Transactions on Information Systems, 26(3), 12.

[20] Pang, B., \& Lee, L. 2008. Opinion mining and sentiment analysis. Foundations and Trends in Information Retrieval, Vol 2 (1-2).

[21] Niu, Y., Zhu, X., Li, J., Hirst, G. 2005. Analysis of polarity information in medical text. In Proceedings of the American Medical Informatics Association 2005 Annual Symposium.

[22] Balahur, A., Kozareva, Z., Montoyo, A. 2009. Determining the polarity and source of opinions expressed in political debates.

[23] Ku, L.W., Li, L.Y., Wu, T.H., Chen, H.H. 2005. Major topic detection and its application to opinion summarization. In Proceedings of the ACM Special Interest Group on Information Retrieval (SIGIR), Salvador, Brasil.

[24] Bansal, M., Cardie, C., Lee, L. 2008. The power of negative thinking: Exploiting label disagreement in the min-cut classification framework. In Proceedings of the International Conference on Computational Linguistics (COLING), 2008. Poster paper, pp.15-18.

[25] Terveen, L., Hill, W., Amento, B., McDonald, D., Creter, J. 1997. PHOAKS: A system for sharing recommendations. Communications of the Association for Computing Machinery (CACM), 40(3), PP. 59-62

[26] Kim, S.M. \& Hovy, E. 2006. Automatic identification of pro and con reasons in online reviews. In Proceedings of the COLING/ACL Main Conference Poster Sessions, pp. 483-490.

[27] Medhat, W., Hassan, A., Korashy, H. 2014. Sentiment analysis algorithms and applications: A survey, Ain Shams Eng.

[28] Mullen, T., \& Collier, N. 2004. Sentiment Analysis using Support Vector Machines with Diverse Information Sources. Proc. of 9th EMNLP, pp. 412-418.

[29] Kudo, T., \& Matsumoto, Y. 2004. A Boosting Algorithm for Classification of Semi-Structured Text. In EMNLP, Vol. 4, pp. 301-308.

[30] Jebaseeli, A. N., \& Kirubakaran, E. 2012. A survey on sentiment analysis of (product) reviews. International Journal of Computer Applications, 47(11).

[31] Kaur, A., \& Gupta, V. 2013. A survey on sentiment analysis and opinion mining techniques. Journal of Emerging Technologies in Web Intelligence, 5(4), 367 371. 
[32] Maynard, D., \& Funk, A. 2011. Automatic detection of political opinions in tweets. In: Proceedings of the 8th international conference on the semantic web, ESWC'11, p. 88-99.

[33] Hu, X., Tang, J., Gao. H., Liu, H. 2013. Unsupervised sentiment analysis with emotional signals. In International Conference on World Wide Web.

[34] Syed, A.Z., Aslam, M., Martinez-Enriquez, A.M. 2014. Associating targets with SentiUnits: a step forward in sentiment analysis of Urdu text. Artificial Intelligence Review, 41(4), pp. 535-561.

[35] Abdulla, N. A., Ahmed, N. A., Shehab, M. A., AlAyyoub, M., Al-Kabi, M. N., \& Al-rifai, S. 2014. Towards improving the lexicon-based approach for arabic sentiment analysis. International Journal of Information Technology and Web Engineering (IJITWE), 9(3), 55-71

[36] Read, J. (2005) Using emoticons to reduce dependency in machine learning techniques for sentiment classification. In ACL Student Research Workshop, pp 43-48

[37] Tausczik, Y.R. \& Pennebaker, J.W. 2010. The psychological meaning of words: Liwc and computerized text analysis methods. Journal of Language and Social Psychology, 29(1):24-54

[38] Dodds, P.S. \& Danforth, C.M. 2009. Measuring the happiness of large-scale written expression: songs, blogs, and presidents. Journal of Happiness Studies, 11(4):441456

[39] Bradley, M.M., Lang, P.J. 1999. Affective norms for English words (ANEW): Stimuli, instruction manual and affective ratings. Technical Report C-1, Gainesville, FL: The Center for Research in Psychophysiology, University of Florida

[40] Thelwall, M., Buckley, K., Paltoglou, G., Cai, D., Kappas, A. 2010. Sentiment strength detection in short informal text. Journal of the American Society for Information Science and Technology, 61(12), pp. 25442558

[41] Bermingham, A., \& Smeaton, A.F. 2010. Classifying Sentiment in Microblogs: Is Brevity an Advantage? In ACM International Conference on Information and Knowledge Management (CIKM), pp. 1833-1836

[42] Paltoglou, G., \& Thelwall, M. 2012. Twitter, MySpace, Digg: Unsupervised Sentiment Analysis in Social Media. ACM Transactions on Intelligent Systems and Technology (TIST), 3(4):66:1-66:19

[43] Esuli, A., \& Sebastiani, F. 2006. Sentiwordnet: A publicly available lexical resource for opinion mining. In Proceedings of LREC Vol. 6, pp. 417-422

[44] Miller, G.A. 1995. WordNet: a lexical database for English. Communications of the ACM, 38(11), 39-41

[45] Gonçalves, P., Benevenuto, F., Cha, M. 2013. Panas-t: A pychometric scale for measuring sentiments on twitter. arXiv preprint arXiv: 1308.1857
[46] Watson, D. \& Clark, L. 1985. Development and validation of brief measures of positive and negative affect: the panas scales. Journal of Personality and Social Psychology, 54(1):1063-1070

[47] Wang, C.J., Tsai, M.F., Liu, T., Chang, C.T. 2013. Financial Sentiment Analysis for Risk Prediction. In Proceedings of the Sixth International Joint Conference on Natural Language Processing pp. 802-808

[48] Cambria, E., Speer, R., Havasi, C., Hussain, A. 2010. SenticNet: A Publicly Available Semantic Resource for Opinion Mining. In AAAI Fall Symposium: Commonsense Knowledge Vol. 10, p. 2 Lecture Notes in Computer Science, 5449,CICLing 2009:468-480

[49] Mayfield, E., \& Rosé, C.P. 2012. LightSIDE: Open Source Machine Learning for Text Accessible to NonExperts. In the Handbook of Automated Essay Grading, Routledge Academic Press

[50] Hassan, A., Abbasi, A., Zeng, D. 2013. Twitter Sentiment Analysis: A Bootstrap Ensemble Framework, Proceedings of the ASE/IEEE International Conference on Social Computing, pp. 357-364

[51] Abbasi , A. 2010. Intelligent Feature Selection for Opinion Classification. IEEE Intelligent Systems, 25(4), pp. 75-79.

[52] Fan, T.K., \& Chang, C.H. 2011. Blogger-centric contextual advertising. Expert Systems with Applications, 38(3), pp. 1777-1788

[53] Qiu, G., He, X., Zhang, F., Shi, Y., Bu, J., Chen, C. 2010. DASA: Dissatisfaction-oriented Advertising based on Sentiment analysis. Expert Systems with Applications, 37 pp.6182-6191

[54] Kang, H., Yoo, S.J., Han, D. 2012. Senti-lexicon and improved Naïve Bayes algorithms for sentiment analysis of restaurant reviews. Expert Systems with Applications ,39 pp. 6000-6010

[55] Cardie, C., Farina, C., Bruce, T., Wagner, E. 2006. Using natural language processing to improve eRulemaking. In Proceedings of Digital Government Research

[56] Kwon, N., Shulman, S., Hovy, E. 2006. Multidimensional text analysis for eRulemaking. In Proceedings of Digital Government Research. In Proceeding of the 2006 international conference on Digital government research, pp. 157-166

[57] Conrad, J.G. \& Schilder, F. 2001. Opinion mining in legal blogs. In Proceedings of the International Conference on Artificial Intelligence and Law (ICAIL), pp. 231-236, New York, NY, USA, 74 ACM

[58] Cao, J., Zeng. K., Wang. H., Cheng, J., Qiao, F., Wen, D., Gao, Y. 2014. Web-Based Traffic Sentiment Analysis: Methods and Applications. ITS(15) 2, April 2014, pp. 844-853

[59] Schumaker, R.P., Zhang, Y., Huang, C.N., Chen, H 2012. Evaluating sentiment in financial news articles". Decision Support Systems 53 pp. 458-464. 